\title{
A Colorimetric Study of Spatial Uniformity in Projection Displays
}

\author{
Jean-Baptiste Thomas $^{1,2}$ and Arne Magnus Bakke ${ }^{1}$ \\ ${ }^{1}$ Gjøvik University College, The Norwegian Color Research Laboratory \\ 2 Université de Bourgogne, Laboratoire d'Electronique Informatique et Image
}

\begin{abstract}
In this paper we investigate and study the color spatial uniformity of projectors. A common assumption is to consider that only the luminance is varying along the spatial dimension. We show that the chromaticity plays a significant role in the spatial color shift, and should not be disregarded, depending on the application. We base our conclusions on the measurements obtained from three projectors. Two methods are used to analyze the data, a conventional approach, and a new one which considers $3 \mathrm{D}$ gamut differences. The results show that the color gamut difference between two spatial coordinates within the same display can be larger than the difference observed between two projectors.
\end{abstract}

\section{Introduction}

Color spatial uniformity for projection displays has been studied[1] 2]. However, it is often considered that only the luminance is of importance, and in most applications only this aspect is corrected for. The chromaticity shift is often considered as negligible. Moreover, the analysis of the color shift along the spatial dimension are mainly supported by either incomplete or qualitative results. This work presents a quantitative analysis of projector spatial nonuniformity. We based our study on two aspects. First a conventional 2D approach is presented, which considers the analysis of a projected full intensity patch. We then use a global comparison of the gamuts at different spatial locations to evaluate the color non-uniformity. We introduce the context and the reasons which have motivated this work in the next section. We then define our experiments, and present the results we obtained. We finally discuss the influence of these results for different applications before we give our conclusions.

\section{Background and Motivation}

A projection system displaying an image on a screen shows some color spatial non-uniformities. These non-uniformities can come from the system properties,

A. Trémeau, R. Schettini, and S. Tominaga (Eds.): CCIW 2009, LNCS 5646, pp. 160-169, 2009.

(C) Springer-Verlag Berlin Heidelberg 2009 
such as lens alignment, but also simply from the position of the projection system compared with the screen. Since CRT displays started being analyzed, it has been widely considered that only the luminance was changing along the spatial dimension [3]. This is still the assumption made by many when modelling newer displays, and they maintain that the chromaticity shift is negligible compared with the change of luminance. In this paper, we demonstrate that the chromaticity shift can not be disregarded, especially for some of todays projection system applications, such as tiled projection systems, and for color research and experiments linked with the human visual system.

Many works have been done in order to characterize the color of projection displays. However, a lot of assumptions used in this case are borrowed from the characterization of CRT monitors. These assumptions have been shown to give a reasonable approximation of the real behavior of this kind of displays [4] [5], but they show their limit with projection displays. Some of these assumptions are already known to be incorrect, such as the non gamma shape of the response curve for LCD systems.

Despite of the studies or tentative works to study or evaluate the color shift along the spatial dimension [1] 2] 6], it is still common to consider that the color varies only in luminance along the spatial dimension of a display. Then it is common in many proposed correction algorithms to only consider a luminance attenuation map, such as in 7] for CRT monitors, and in [8] for projectors or multi-projector systems corrections.

In her study about multi-projector systems, Majumder et al. assessed that the spatial chromaticity shift is negligible compared to the luminance shift. However, looking at the figures presented in 9, the gamut shows a severe shift, which at first seems to be comparable to the difference observed from one display to a completely different one.

While Majumder et al. looked at the projector gamuts in chromaticity diagrams, Bakke et al. [10] recently proposed a method for computing the difference between two gamuts in a 3-D color space. They suggested that a method using discretized representations of the gamuts can be used to compute the relative gamut mismatch between two gamut boundaries.

First, a binary voxel structure is created for each gamut. The value of each position is determined using the following method. If the position is within the gamut, the value is set to one, otherwise it is set to zero. Determining the differences between two gamuts can then be simplified to counting the positions where the values of the two gamut representations are different, and multiplying this count with the volume of the cube represented by a single discretized position. The resulting number can be divided by the volume of the reference gamut, giving the relative gamut mismatch.

\section{Experimental Setup}

We performed our investigation on three displays, two LCD projectors from the same model and manufacturer (Sony VPL-AW 15), and one DLP projector 
(Projection Design Action One). They are named LCD1, LCD2 and DLP in the following. All the displays were used with the default settings. In order to have accurate measurements, we used the CS-1000 spectroradiometer from Minolta. The measurements were done in a dark surrounding, so that no light is involved except that from the display. A warming up time of at least one hour and fifteen minutes has been used before any measurement to reach a correct temporal stability. The geometry of the all system was basically of the same type that the one used in [2].

In our first experiment, we used the same kind of approach as is described in the IEC draft [6] and in the work of Kwak and MacDonald [2]. We measured only a full intensity white image $(\mathrm{RGB}=[255,255,255])$ at $5 \times 5$ locations regularly spread along the display, having positioned the measurement devices in approximately the same position as described in [2].

In addition to this approach, we are interested in looking at the differences in the gamut volume of the projector. We chose to limit the measurement process to 9 spatial positions among the set of 25, because of the time needed to complete the measurements. Bakke [10] showed that the gamut boundaries descriptor algorithm suggested by Balasubramanian and Dalal [11] performs well on most data sets. We have therefore used the modified convex hull with a preprocessing step using $0.2 \gamma$ to compute the gamuts. In order to perform the gamut evaluation, we used the ICC3D framework [12].

The evaluation is performed in the CIELAB color space. We encountered a challenging issue in using this space. In the past studies we know, since the luminance was supposed to be at its highest value in the center of the display and since the observer was supposed to look at the center first, the measurement of a white patch at this position was used as the reference white. This follows the recommendation of the IEC draft [6]. However, considering the position of the display or the alignment of the lens, the highest luminance point can be severely shifted from the center. That can happen for instance when the projector is made to be used in an office and to project the image on a wall for presentation, such as the DLP projector we tested.

We decided then to use the brightest point of the white image displayed as reference white. This choice has some advantages in our case. If we consider the geometry of the system and the lens alignment, choosing the reference white at the highest point is more in accordance with the physical properties of the device. Since we base our experiment on colorimetry, and we do not to attempt to take more human factors into consideration, we have chosen to use this as our reference white.

In the following, we call global reference white the measurement of the brightest white, and local reference white, the white measured at the different locations.

\section{Results}

In this section we present and discuss the results we obtained, first with the conventional evaluation, secondly with the 3D gamut comparison approach. 

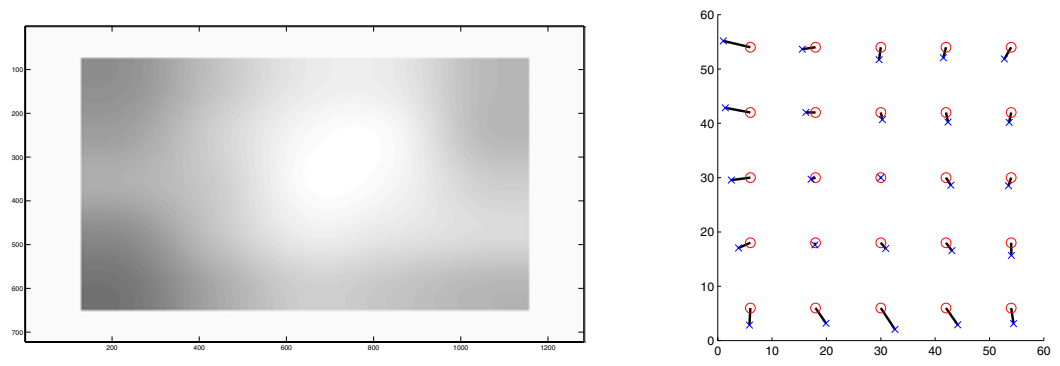

(a) Lightness, chroma and hue shift for display LCD1
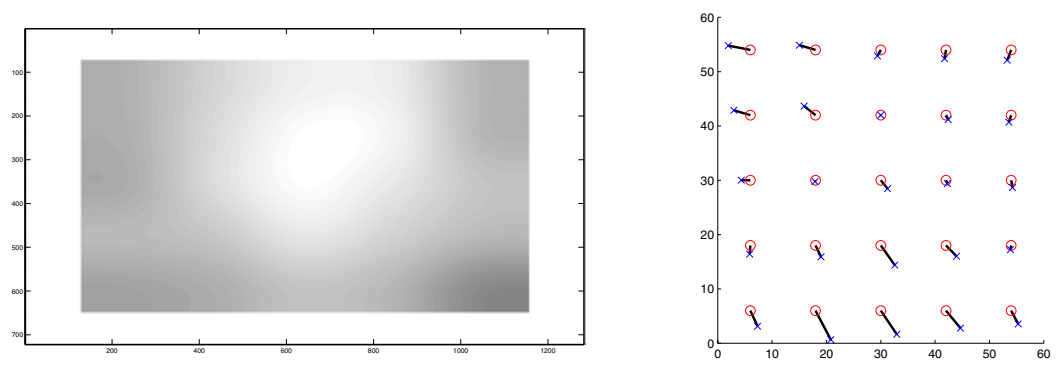

(b) Lightness, chroma and hue shift for display LCD2
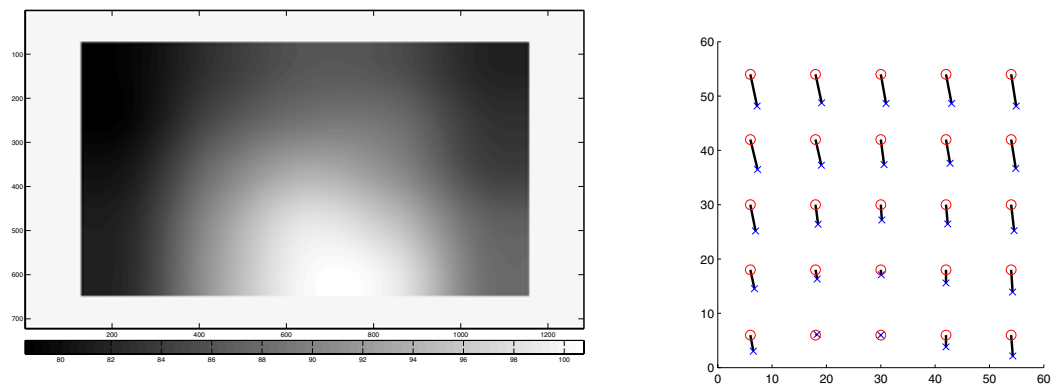

(c) Lightness, chroma and hue shift for display DLP

Fig. 1. Visualization of the color shift throughout the display. On the left, we show a visualization of the lightness shift. The maximum lightness is 100 (white), the minimum (black) is around 79. On the right, hue and chroma shift are plotted relative to their spatial position. The position of the circles is the reference, the crosses indicate the measured value. The angle of the segment represent the hue shift, and the norm the chroma shift in the $\left(a^{*}, b^{*}\right)$ plane. 
Table 1. Relative shift in lightness and chroma at 25 locations for the three tested displays

\begin{tabular}{|c|c|c|c|c|c|c|c|c|c|c|c|}
\hline \multicolumn{6}{|c|}{ Shift in lightness } & \multicolumn{6}{|c|}{ Shift in Chroma } \\
\hline \multicolumn{12}{|c|}{ LCD1 } \\
\hline & 1 & 2 & 3 & 4 & 5 & $\Delta$ & 1 & 2 & 3 & 4 & 5 \\
\hline 1 & -8.92 & 4.85 & -1.61 & -1.60 & -5.55 & & 5.09 & 2.46 & 2.29 & 1.99 & 2.49 \\
\hline 2 & -7.66 & 3.72 & -0.37 & -0.36 & -5.55 & 2 & 4.68 & $\mid 1.78$ & 1.36 & 1.81 & 1.97 \\
\hline 3 & -6.42 & 4.09 & 0.00 & -0.58 & -3.74 & 3 & 3.53 & 0.87 & 0.00 & 1.65 & 1.56 \\
\hline 4 & -9.29 & .77 & -1.29 & -1.91 & -2.81 & 4 & 2.37 & 0.40 & 1.39 & 1.80 & 2.31 \\
\hline 5 & -11.27 & -7.02 & -3.78 & -4.64 & -5.84 & 5 & 3.16 & 3.41 & 4.73 & 3.77 & 1.91 \\
\hline \multicolumn{12}{|c|}{ LCD2 } \\
\hline$L^{*}$ & 1 & 2 & 3 & 4 & 5 & $\Delta C^{*}$ & 1 & 2 & 3 & 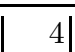 & \\
\hline 1 & -6.49 & -3.43 & -1.14 & -1.53 & -6.09 & 1 & 4.13 & 3.09 & 1.26 & 1.63 & 2.03 \\
\hline 2 & -6.63 & \begin{tabular}{l|}
-2.93 \\
\end{tabular} & 0.00 & -0.90 & -5.96 & 2 & 3.17 & 2.68 & 0.00 & 0.92 & 1.38 \\
\hline 3 & -6.90 & -2.85 & -0.11 & -2.00 & -4.78 & 3 & 1.67 & 0.24 & 1.97 & $\mid 0.66$ & 1.35 \\
\hline 4 & -5.71 & \begin{tabular}{l|}
-4.68 \\
\end{tabular} & -1.94 & -3.79 & -5.89 & 4 & 1.60 & \begin{tabular}{|l|}
2.32 \\
\end{tabular} & 4.44 & \begin{tabular}{|l|}
2.77 \\
\end{tabular} & 0.78 \\
\hline 5 & -7.59 & -6.75 & -4.82 & -6.09 & -9.66 & 5 & 3.18 & 6.03 & 5.25 & 4.18 & 2.76 \\
\hline \multicolumn{12}{|c|}{$\overline{\mathrm{DLP}}$} \\
\hline$L^{*}$ & 1 & 2 & 3 & 4 & 5 & $\Delta C^{*}$ & 1 & 2 & 3 & 4 & \\
\hline 1 & \begin{tabular}{|l|}
-20.88 \\
\end{tabular} & \begin{tabular}{|l|}
-16.72 \\
\end{tabular} & -13.84 & -14.40 & -18.14 & 1 & 5.97 & 5.37 & 5.47 & 5.47 & 5.92 \\
\hline 2 & -20.90 & \begin{tabular}{|c|}
-14.79 \\
\end{tabular} & -11.49 & -11.83 & -16.80 & 2 & 5.68 & 4.85 & 4.65 & 4.44 & 5.40 \\
\hline 3 & -19.39 & \begin{tabular}{|l|}
-11.46 \\
\end{tabular} & -6.63 & -9.29 & -15.60 & 3 & 4.94 & 3.62 & 2.81 & 3.56 & 4.81 \\
\hline 4 & -18.06 & -8.61 & -1.68 & -4.87 & -12.63 & 4 & 3.53 & \begin{tabular}{|l|}
1.70 \\
\end{tabular} & \begin{tabular}{|l|}
0.92 \\
\end{tabular} & 2.41 & 4.09 \\
\hline 5 & -17.77 & \begin{tabular}{l|}
-7.62 \\
\end{tabular} & 0.00 & -1.21 & -11.58 & 5 & 3.01 & 0.31 & 0.00 & 2.18 & 3.85 \\
\hline
\end{tabular}

\subsection{Conventional Evaluation}

By displaying the white patch and measuring the projected color at each position, we get an overview of the global behavior of the display. In Figure 1, we can see the lightness shift along the spatial dimension in the left part of the figure. This visualization is based on the measurements at 25 locations. The white surround comes from the fact that we have no information on this part of the displayed area, while we can interpolate the data inside this rectangle. We can see that the brightness point is not necessarily in the center of the screen. The color shift is illustrated in the right part of this figure. We can see the same effect as the one described in 2, a shift in the color around the center of the lens displayed on the screen (i.e., the brightest point). The LCDs projectors show a shift from green/cyan to blue/red as a general behavior from the top left corner to the bottom right. The DLP shows a shift to the blue from the top to the bottom. The causes of this shift can be found in the literature 13 . 
Table 2. Relative shift in CIELAB unit at 25 locations for the three tested displays

\begin{tabular}{|c|c|c|c|c|c|}
\hline \multicolumn{6}{|c|}{ Shift in CIELAB unit } \\
\hline \multicolumn{6}{|c|}{ LCD1 } \\
\hline$E_{a b}^{*}$ & 1 & 2 & 3 & 4 & 5 \\
\hline 1 & 9.26 & 5.24 & 2.80 & 2.93 & 7.53 \\
\hline 2 & 7.89 & 4.14 & 1.41 & 1.81 & 7.26 \\
\hline 3 & 6.61 & 4.41 & 0.00 & 1.05 & 5.14 \\
\hline 4 & 9.57 & 5.10 & 1.89 & 1.95 & 3.68 \\
\hline 5 & 11.64 & 7.97 & 6.05 & 5.75 & 6.64 \\
\hline \multicolumn{6}{|c|}{$\overline{\mathrm{LCD} 2}$} \\
\hline$\Delta E_{a b}^{*}$ & 1 & 2 & 3 & 4 & \\
\hline 1 & 6.80 & 3.80 & 1.70 & 3.45 & 7.36 \\
\hline 2 & 6.77 & 3.07 & 0.00 & 2.82 & 6.75 \\
\hline 3 & 7.03 & 2.92 & \begin{tabular}{|c|}
1.97 \\
\end{tabular} & 2.02 & 5.07 \\
\hline 4 & 5.76 & 5.44 & 4.84 & 4.44 & 6.11 \\
\hline 5 & 8.07 & 7.94 & 7.13 & 8.57 & 10.17 \\
\hline \multicolumn{6}{|c|}{$\overline{\mathrm{DLP}}$} \\
\hline$\Delta E_{a b}^{*}$ & 1 & 2 & 3 & 4 & \\
\hline 1 & 21.71 & \begin{tabular}{|l|l|}
17.60 & 1 \\
\end{tabular} & 14.88 & 15.36 & 19.09 \\
\hline 2 & 21.58 & 15.45 & 12.40 & 12.78 & 17.73 \\
\hline 3 & 19.97 & 12.00 & 7.20 & 9.97 & 16.36 \\
\hline 4 & 18.52 & 8.94 & 1.92 & 5.16 & 13.11 \\
\hline 5 & 18.18 & 7.92 & 0.00 & 1.25 & 11.97 \\
\hline
\end{tabular}

The results of the quantitative analysis are presented in Tables 1 and 2, The first shows the $\Delta L^{*}$ and $\Delta C^{*}$ relative to the brightest point. The second shows the $\Delta E_{a b}^{*}$.

The largest $\Delta E_{a b}^{*}$ observed are 11.64, 10.17 and 21.71 for LCD1, LCD2 and DLP respectively. The differences are definitely over the noticeable difference from a colorimetric point of view.

For the LCDs, we noticed a maximum lightness shift of 11.27 units in the bottom left corner for LCD1, and of 9.66 units in the bottom right corner for LCD2. The corresponding chroma shifts are respectively of 3.16 and 2.76. The maximum chroma shifts for these displays are 5.09 in the upper left corner for LCD1 and 6.03 at the bottom left for LCD2, with associated lightness shifts of 8.92 and 6.75. The DLP projector shows a maximum lightness shift of 20.90 units in the upper left part of the displayed area, and 5.68 units in chroma at the same position. The maximum chroma shift is of 5.97 units in the upper left corner for 20.88 units in lightness.

In some locations we can clearly see that the lightness variation is smaller than or equivalent to the chromaticity shift, such as below the center for LCD2, which shows a $\Delta L^{*}$ of 1.94 and a $\Delta C^{*}$ of 4.44 compared to the reference location. 
Table 3. Relative gamut mismatch for each position compared with the gamut of the position with the highest luminance. The gamuts are calculated using the global white point as well as the local white point for each of the 9 selected locations.

\begin{tabular}{|c|c|c|c|c|c|c|c|}
\hline \multicolumn{4}{|c|}{ Gamut mismatch, global white point } & \multicolumn{4}{|c|}{ Gamut mismatch, local white point } \\
\hline \multicolumn{8}{|c|}{ LCD1 } \\
\hline$\%$ & 1 & 3 & 5 & $\%$ & 1 & 3 & 5 \\
\hline 1 & 27.23 & 4.90 & 17.08 & 1 & 9.57 & 3.30 & 5.72 \\
\hline 3 & 23.92 & 0.00 & 16.15 & 3 & 7.49 & 0.00 & 5.53 \\
\hline 5 & 32.66 & 9.48 & 13.50 & 5 & 7.90 & 2.07 & 4.09 \\
\hline \multicolumn{8}{|c|}{ LCD2 } \\
\hline$\%$ & 1 & 3 & 5 & $\%$ & 1 & 3 & 5 \\
\hline 1 & 24.84 & 5.83 & 19.75 & 1 & 9.42 & 2.48 & 4.46 \\
\hline 3 & 20.18 & 0.00 & 18.79 & 3 & 6.00 & 0.00 & 2.40 \\
\hline 5 & 29.75 & 11.01 & 20.82 & 5 & 5.98 & 1.98 & 2.48 \\
\hline \multicolumn{8}{|c|}{$\overline{\mathrm{DLP}}$} \\
\hline$\%$ & 1 & 3 & 5 & $\%$ & 1 & 3 & 5 \\
\hline 1 & 52.36 & 38.02 & 41.06 & 1 & 8.51 & 6.86 & 6.91 \\
\hline 3 & 47.73 & 18.29 & 36.28 & 3 & 7.96 & \begin{tabular}{|c|}
3.92 \\
\end{tabular} & 6.38 \\
\hline 5 & 43.22 & 0.00 & 26.93 & 5 & 6.62 & 0.00 & 4.87 \\
\hline
\end{tabular}

When we consider the hue shift which is shown in Figure 1 on the right, the chromaticity difference from a spatial coordinate to another can easily be larger than the lightness shift, and the hypothesis which considers the color shift as negligible can be disputed.

\subsection{D Gamut Evaluation}

The reference gamut for each projector was constructed from the measurement data of the position with the highest luminance value. Table 3 contains the percentage of gamut mismatch for each position compared with this reference.

As we can see, the gamut at some locations can be as much as $52 \%$ smaller than the reference, which is illustrated in Figures 2 a and $2 \mathrm{r}$. The luminance shift is responsible for a large part of this difference, but compensating for the luminance shift by using the local white point for calculating CIELAB values still leaves a significant maximum gamut mismatch of $8.51 \%, 9.42 \%$ and $9.57 \%$ for the three projectors. Figures $2 \mathrm{~b}$ and $2 \mathrm{~d}$ show the gamuts computed using the local white point.

This mismatch is comparable in relative volume to the error introduced when using a strictly convex hull to represent the gamut of an arbitrarily chosen device, 


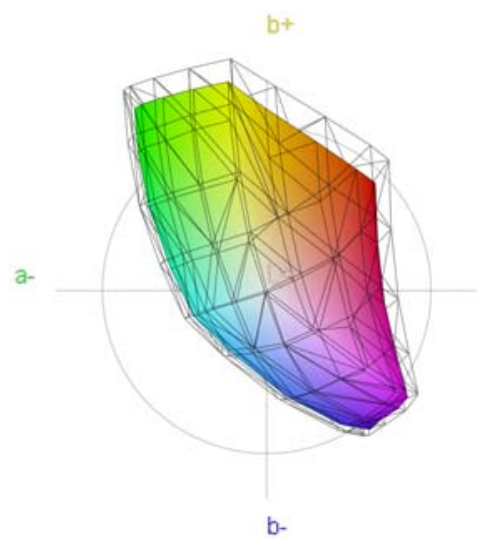

(a) LCD1, global white point

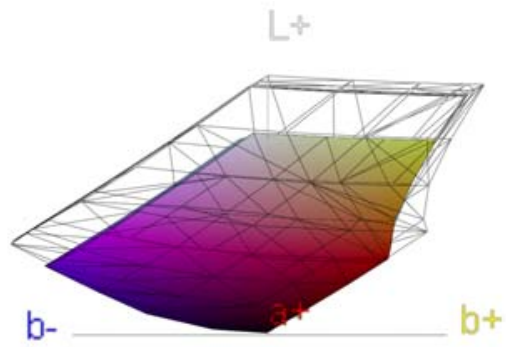

(c) DLP, global white point

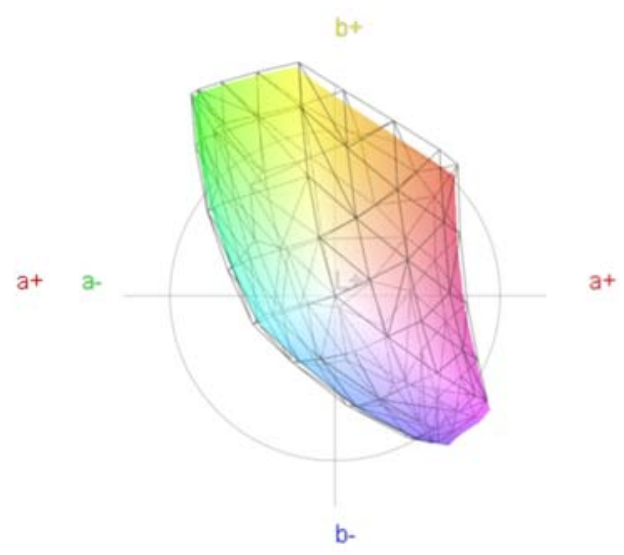

(b) LCD1, local white point

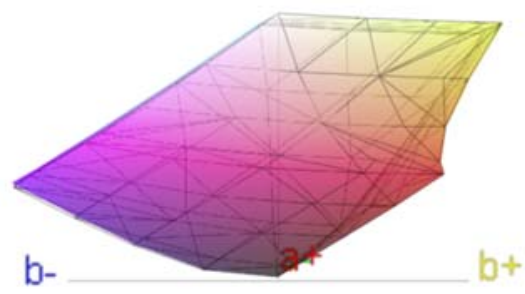

(d) DLP, local white point

Fig. 2. The gamut boundaries for two of the projectors at the position with the highest luminance (wireframe) compared with the gamut of the top left corner (solid and wireframe). CIELAB measurement values were computed relative to the global white point for (a) and (c) while (b) and (d) utilizes the white point of each location.

and is greater than many inter-device gamut differences. In our experiment, the gamut mismatch between the two LCD projectors (at the reference position) is $2.75 \%$, giving an intra-device difference 3.43 times larger than the inter-device difference.

The DLP shows large differences in gamut depending on the spatial location, similar to what we showed in our analysis of lightness. Compared with the two LCDs, a larger part of the differences can be explained by the luminance shift. The remaining gamut mismatch volume mainly consists of the volume that is contained within the reference and is not a part of the gamut of the other spatial locations, which is illustrated in Figure 3. This means that there are effects in addition to the luminance shift which contribute to the reduction of the gamuts. 


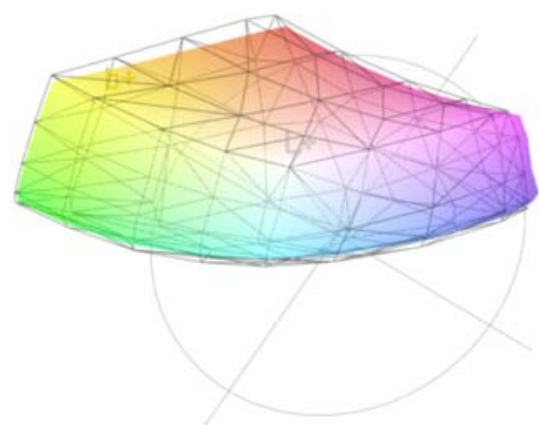

(a) LCD1

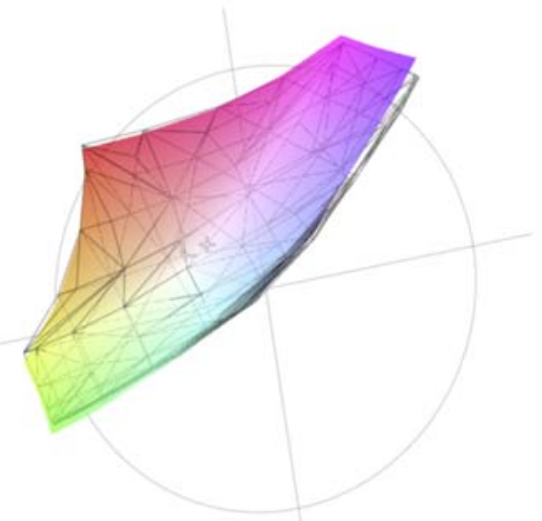

(b) DLP

Fig. 3. While using the white point of each location reduces the difference between the gamuts by compensating for the luminance shift, we still see some difference between the gamuts

\section{Discussion}

Based on our analysis of these results, there appears to be sufficient evidences to claim that the chromaticity shift has to be taken into account in some cases. Some applications might not be affected, while some might suffer seriously from this fact. It appears important for us to compensate for this problem in at least two situations. While performing psychophysical experiments for color science purpose with a projector, and while tiling projectors together to build a multiprojector system.

Related to the choice we made in our experiment by using the brightest white point as a reference, we found that the gamut of the position with the largest luminance results in the largest estimated gamut volume. It is then a logical choice to use this as the basis for the reference gamut.

Considering the case of a multi-projector systems, since the chroma is shifting in two opposite hue directions from the center of the lens, the area around the overlapping edges will show two really different colors. Note that even if the computed chrominance shift is major, since we observed some $\Delta C^{*}$ of about 6 from a position to another and greater differences can be found between extreme positions, if we consider the spatial content of an image, it is not certain that the chrominance shift will break the perceived uniformity.

Similarly, the reduction in gamut volume of up to $52 \%$ when using the global white point does not appear to be indicative of the perceived color capability of the projectors. However, using the local white point seems to underestimate the real difference. This is indorsed by the conventional approach. When we look at the full intensity white patch, the perceived difference does not seem to be as large as the measured one. 
In order to make a model which fits our perceived color appearance, we need to consider more psychovisual features, such as the color adaptation at the local and at the global level, cognition and physiology.

\section{Conclusion}

We have shown that the measured chromaticity shift along a projector is important, and that considering only the luminance as non-uniform can be a critical mistake in some applications. However, considering the image content, it is reasonable to think that the perceived non-uniformity would not be broken. Further experiments could be done in this direction to find what can be considered as perceived spatial uniformity. As a straightforward continuation of this work, we think it could be of great interest to utilize spatial gamut algorithm using a spatially varying gamut in multi-projector systems.

\section{References}

1. Seime, L., Hardeberg, J.Y.: Colorimetric characterization of LCD and DLP projection displays. Journal of the Society for Information Display 11(2), 349-358 (2003)

2. Kwak, Y., MacDonald, L.: Characterisation of a desktop LCD projector. Displays 21(5), 179-194 (2000)

3. Brainard, D.H., Pelli, D., Robson, T.: Display characterization, Encyclopedia of Imaging Science and Technology. Wiley, New-York (2002)

4. Cowan, W., Rowell, N.: On the gun independency and phosphor constancy of color video monitor. Color Research \& Application 11, S34-S38 (1986)

5. Berns, R.S., Gorzynski, M.E., Motta, R.J.: CRT colorimetry. part II: Metrology. Color Research \& Application 18(5), 315-325 (1993)

6. IEC:61966-6: Color measurement and management in multimedia systems and equipment, part 3: Equipment used for digital image projection, committee Draft (August 1998)

7. Brainard, D.H.: Calibration of a computer-controlled color monitor. Color Research \& Application 14, 23-34 (1989)

8. Majumder, A., Stevens, R.: Lam: Luminance attenuation map for photometric uniformity in projection based displays. In: Proceedings of ACM Virtual Reality and Software Technology, pp. 147-154 (2002)

9. Majumder, A., Stevens, R.: Color nonuniformity in projection-based displays: Analysis and solutions. IEEE Transactions on Visualization and Computer Graphics 10(2), 177-188 (2004)

10. Bakke, A.M., Hardeberg, J.Y., Farup, I.: Evaluation of gamut boundary descriptors, 50-55 (2006)

11. Balasubramanian, R., Dalal, E.: A method for quantifying the color gamut of an output device. In: Color Imaging: Device-Independent Color, Color Hard Copy, and Graphic Arts II, January 1997, vol. 3018. SPIE, San Jose (1997)

12. Farup, I., Hardeberg, J.Y., Bakke, A.M., le Kopperud, S., Rindal, A.: Visualization and interactive manipulation of color gamuts, 250-255 (2002)

13. Matthew, M., Brennesholtz, S., Stupp, E.H.: Projection Displays, 2nd edn. John Wiley \& Sons, Ltd., Chichester (2008) 